\title{
Dissociative electron attachment to HBr: A temperature effect
}

\author{
J. Fedor, ${ }^{*}$ M. Cingel, J. D. Skalný, ${ }^{\dagger}$ P. Scheier, and T. D. Märk ${ }^{\ddagger}$ \\ Institut für Ionenphysik und Angewandte Physik, Leopold-Franzens Universität, Technikerstrasse 25, A-6020 Innsbruck, Austria \\ M. Č́ížek, P. Kolorenč, and J. Horáček \\ Institute of Theoretical Physics, Faculty of Mathematics and Physics, Charles University Prague, V Holešovičkách 2, 18000 Praha 8 , \\ Czech Republic
}

\begin{abstract}
The effects of rovibrational temperature on dissociative electron attachment to hydrogen bromide has been investigated from the experimental and theoretical point of view. Theoretical calculations based on the nonlocal resonance model predict a strong temperature effect on the $\mathrm{Br}^{-}$fragment ion yield due to population of higher vibrational and rotational states. A crossed beam experimental setup consisting of a temperature controlled effusive molecular beam and a trochoidal electron monochromator has been used to confirm this prediction. The high degree of agreement between experiment and theory indicates the validity of the theoretical model and its underlying physical picture.
\end{abstract}

PACS number(s): $34.80 . \mathrm{Ht}$

\section{INTRODUCTION}

Hydrogen halides constitute an ideal system for testing theoretical models for electron-molecule collisions: recent results for $\mathrm{HF}[1], \mathrm{HCl}[2,3]$, and $\mathrm{HBr}[4]$ demonstrated that hydrogen halides provide much richer variety of resonance phenomena than the long-time prototype system $\mathrm{N}_{2}$. They include pronounced threshold peaks in the vibrational excitation cross section, steplike features (Wigner cusps) in the dissociative electron attachment (DEA) cross section and distinctly different shapes of the DEA cross section for different vibrational and rotational levels of the neutral molecule. The latter feature leads to clearly observable temperature effects.

Among hydrogen halides, the electron- $\mathrm{HBr}$ collision system has received much less attention than, for example, the electron- $\mathrm{HCl}$ system. The first measurement of the dissociative electron attachment $\mathrm{Br}^{-} / \mathrm{HBr}$ ion yield is due to Schulz and co-workers [5]. Abouaf and Teillet-Billy [6] used a better electron energy resolution and reported (i) a structure in the cross section shape at about $0.3 \mathrm{eV}$ (the low energy part of the main DEA peak) and (ii) sharp drops in the cross section on the high energy side of the main peak.

The most comprehensive experimental study of electron$\mathrm{HBr}$ collisions at $T=310 \mathrm{~K}$ was presented by Allan and coworkers [4]. Using a high electron energy resolution apparatus with an energy spread of about $15 \mathrm{meV}$, the authors measured relative cross sections for the vibrational excitation and for the dissociative electron attachment. They identified the structure at approximately $0.3 \mathrm{eV}$ to be a separated peak,

\footnotetext{
*Present address: Department of Chemistry, University of Fribourg, Chemin du Musee 9, 1700 Fribourg, Switzerland.

${ }^{\dagger}$ Permanent address: Department of Experimental Physics, Comenius University, Mlynska dolina F2, 84215 Bratislava, Slovakia.

${ }^{\ddagger}$ Also adjunct professor at Department of Experimental Physics, Comenius University, Mlynska dolina, 84215 Bratislava, Slovakia. Electronic address tilmann.maerk@uibk.ac.at
}

however, having a lower relative intensity than observed by Abouaf and Teillet-Billy [6].

The experimental research on low-energy electron scattering from $\mathrm{HBr}$ and hydrogen halides in general has been accompanied by intense theoretical efforts over many years. A variety of theoretical concepts and methods have been developed to explain the experimental findings. A complete survey of the theoretical developments can be found in reviews by Morrison [7], Fabrikant [8], Domcke [9], and Horáček [10].

As to the role of the vibrational and rotational excitation in the DEA process, early experiments on selected molecules (e.g., $\mathrm{O}_{2}, \mathrm{~N}_{2} \mathrm{O}, \mathrm{CO}_{2} \ldots$ ) $[11,12]$ have shown a large increase in cross section and decrease in threshold energy with temperature. The first detailed theoretical study by O'Malley [13] on $\mathrm{O}^{-} / \mathrm{O}_{2}$ yielded good agreement with experiments $[14,15]$ and explained the temperature shift by the effect of the rapidly varying survival probability. The first study to resolve the contribution from specific vibrational and rotational states was due to Allan and Wong [16] on $\mathrm{H}^{-} / \mathrm{H}_{2}$ production. This DEA process has been subsequently extensively theoretically analyzed, e.g., in Refs. [17-20]. Vibrationally resolved DEA data exist also for $\mathrm{HCl}$ and $\mathrm{HF}$ [21]. The DEA temperature dependence has also been studied in a variety of complex molecules, including $\mathrm{SF}_{6}[22,23], \mathrm{SF}_{5} \mathrm{Cl}$ [23], $\mathrm{SO}_{2} \mathrm{~F}_{2}$ [24], and a number of halogenated compounds, e.g., Refs. [25-27]. An alternative preparation of the vibrationally excited molecules via Franck-Condon pumping has been utilized in the DEA study to $\mathrm{Na}_{2}$ by Külz et al. [28]

The adequacy of the nonlocal resonance model in describing cross sections for DEA to hydrogen halides at room temperature has been demonstrated previously $[1,3,4]$. Moreover, the nonlocal resonance model has proven capable to recover strong interchannel coupling effects, experimentally observed for the associative detachment process in $\mathrm{Cl}^{-}+\mathrm{H}$ and $\mathrm{Br}^{-}+\mathrm{H}$ [29], i.e., for the inverse process of DEA, closely connected with DEA by microscopic reversibility. However, there is a disagreement between predictions of this model and the experimental results for DEA to vibrationally and rotationally excited $\mathrm{HCl}$. The calculated cross sections for the production of $\mathrm{Cl}^{-} / \mathrm{HCl}$ [3] at different temperatures of 
the neutral gas were compared with the experimental data of Allan and Wong [21]. The position of the structures in the ion yield spectrum agreed quite well, but their relative height differed significantly at different temperatures. It was concluded [3] that a systematic error in the measurement of the temperature of Allan and Wong might have been responsible for the discrepancy between theory and experiment.

The scope of the present work was to perform analogous measurements for $\mathrm{HBr}$ and compare with the predictions of the nonlocal resonance model. We report experimental results for $\mathrm{DEA}$ to $\mathrm{HBr}$ in the gas temperature range of 310 to $870 \mathrm{~K}$. The measurements were performed utilizing a newly designed temperature controlled effusive molecular beam source in combination with a high resolution trochoidal electron monochromator. In the theoretical part we report calculations of the DEA cross section to different vibrational and rotational states of $\mathrm{HBr}$ within a nonlocal resonance model. To allow for a detailed comparison between experiment and theory, the theoretical data were appropriately averaged over the rotational and vibrational distribution of the target gas and convoluted with an experimental resolution function. The comparison of the resulting cross sections with experiment reveals a high degree of agreement.

\section{EXPERIMENT}

The trochoidal electron monochromator (TEM) combined with a quadrupole mass spectrometer used in this study was described previously [30]. The quasimonoenergetic electron beam was crossed in the shielded TEM collision cell at $90^{\circ}$ with an effusive beam of neutral molecules. The molecular beam source consists of a stainless steel cylinder ended by a capillary embedded in the cylinder. The capillary has a length of $3 \mathrm{~cm}$ and a diameter of $0.5 \mathrm{~mm}$. The distance between the end of the capillary and the TEM collision chamber was approximately $1 \mathrm{~cm}$. The gas pressure in the cylinder was kept around 1 mbar, the corresponding pressure in the monochromator chamber was of the order of $1 \times 10^{-5}$ mbar. These parameters assured that the character of the flow through the capillary was molecular [31] and that the collisions of the molecules with the surface were the dominant collision process in the capillary. These conditions have to be fulfilled in order to control the vibrational and rotational temperature of the neutral molecules prior to DEA. The gas cylinder was heated by five pairs of tungsten wires and the temperature was measured inside the cylinder at the inner end of the capillary via a PT100 sensor. The temperature range in the present experiment was 310 to $870 \mathrm{~K}$.

We assume that radiative cooling of the vibrationally and rotationally excited molecules prior to the DEA collisions can be neglected. For example, the dipole transition moment for $\mathrm{HBr} v=1 \rightarrow 0$ IR emission is 0.036 Debye [32] and the corresponding natural lifetime for this emission is 0.14 seconds [33].

The electron energy scale was calibrated using the strong $s$-wave resonance in $\mathrm{SF}_{6}$ [34], and the broadening of the $\mathrm{SF}_{6}^{-}$ peak also reflects the electron energy resolution (see discussion below). The energy resolution at very low electron energies estimated from the width of the $\mathrm{SF}_{6}^{-}$peak was
$35 \mathrm{meV}$, however, at higher electron energies $(0.5-1 \mathrm{eV})$ the electron energy resolution decreases to approximately $50 \mathrm{meV}$ as was shown by the retarding field analysis.

The anions produced were mass analyzed in a quadrupole mass filter and detected in the channeltron type secondary electron multiplier. No difference in the ion yield spectra for two bromine isotopes ${ }^{79} \mathrm{Br}^{-}$and ${ }^{81} \mathrm{Br}^{-}$was observed, the quadrupole mass resolution was thus decreased to merge the two masses in order to obtain an increased ion signal.

\section{THEORETICAL APPROACH}

The dissociative electron attachment was described within the nonlocal resonance formalism [9]. This model is based on the assumption that a temporary molecular negative-ion state (resonance) is formed during the collision process and that this resonance accounts for the coupling of the electronic scattering dynamics with the nuclear motion. The resonance is represented by a square-integrable discrete state $\left|\varphi_{d}\right\rangle$ which interacts with a continuum of scattering states $\left|\varphi_{\epsilon}\right\rangle$ via coupling matrix elements $V_{d \epsilon}$. The scattering problem was reduced to a problem of solving nuclear dynamics in one dimension using a projection operator approach. The DEA $T$ matrix and the cross section were determined by the corresponding matrix elements between nuclear wave functions. The nuclear dynamics is fully determined by the potential of the neutral molecule $V_{0}(R)$, the potential of the discrete negative ion state $V_{d}(R)$ and the coupling matrix elements $V_{d \epsilon}(R)$.

An essential ingredient of the nonlocal resonance model especially for hydrogen halides is the explicit consideration of threshold effects induced by the long-range dipole potential. The dipole-induced nonanalyticities of the $S$ matrix and related quantities at threshold enter through the threshold expansion of the energy dependent width function

$$
\Gamma(\epsilon)=2 \pi\left|V_{d \epsilon}\right|^{2}
$$

and the associated level shift [9]. Details of this model have been given elsewhere $[3,4]$.

Using this approach cross sections for DEA to individual vibrational $v$ and rotational $J$ states were calculated. To compare with the experimental data, Boltzmann population of these states was assumed and the cross section at the temperature $T$ was calculated as

$$
\sigma(\epsilon, T)=\frac{\sum_{v, J} \sigma_{v, J}(\epsilon)(2 J+1) e^{-E_{v, J} / k T}}{\sum_{v, J}(2 J+1) e^{-E_{v, J} / k T}},
$$

where $E_{v, J}$ is the excitation energy of the $(v, J)$ level of the neutral molecule and $k$ is the Boltzmann constant. The summation was taken over vibrational and rotational quantum numbers up to $v_{\max }=8$ and $J_{\max }=50$. The calculated cross sections were further convoluted with the electron energy distribution function $f(\epsilon)$ as

$$
\sigma(E, T)=\int_{\epsilon>0} \sigma(\epsilon, T) f(\epsilon-E) d \epsilon
$$

to allow for realistic comparison with the raw experimental data. 


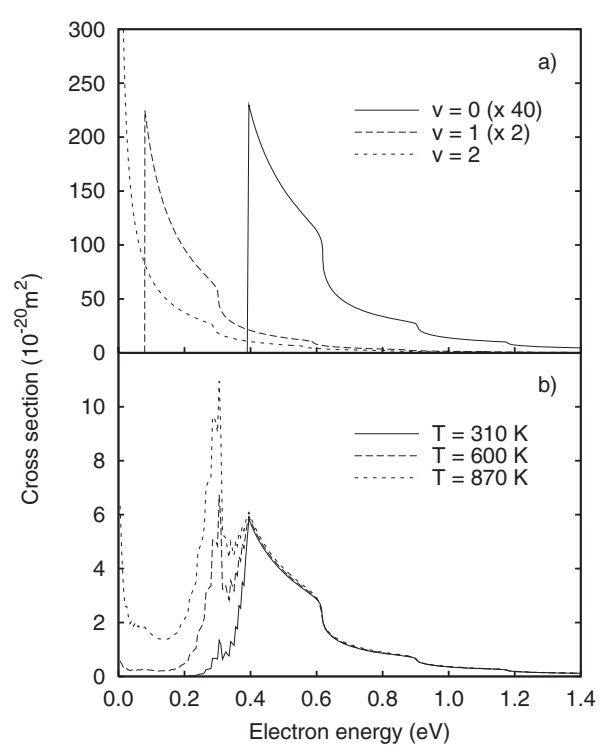

FIG. 1. Theoretical cross section for the DEA to $\mathrm{HBr}$ for (a) different vibrational states of the $\mathrm{HBr}$ molecule in $J=0$ rotational state and (b) rotovibrational population corresponding to the Maxwell-Boltzmann distribution at different temperatures.

\section{RESULTS AND DISCUSSION}

The calculated cross sections for DEA to the HBr molecule in its first three vibrational states are shown in Fig. 1(a). Predictions of the DEA cross section for different temperatures of the neutral molecule are shown in Fig. 1(b). All cross sections exhibit steplike features on the high energy side (Wigner cusps). These sudden decreases of the $\mathrm{Br}^{-}$production yield can be explained on the basis of strong competition between autodetachment and dissociation of the transient negative ions $(\mathrm{HBr})^{*-}[6]$. Their positions correspond to the opening of the $0 \rightarrow 2,0 \rightarrow 3$, and $0 \rightarrow 4$ vibrational excitation channels in the neutral $\mathrm{HBr}$. The vibrational excitation process has a steep onset and the channel interaction produces the cusp structure in the DEA cross section. Two additional peaks are growing at elevated temperatures. The peak at $0.306 \mathrm{eV}$ is due to molecules in the ground vibrational, but rotationally excited state, $J \sim 10$. The cross section for this particular rotational state is enhanced at threshold due to the Wigner cusp at the opening of the 0 $\rightarrow 1$ vibrational excitation channel. The peak growing at zero energy at temperatures above $600 \mathrm{~K}$ contains significant contributions from molecules in the first excited vibrational state $v=1$ because for $J \geqslant 8$ the DEA process becomes exothermic (the DEA cross section diverges at zero energy).

Moreover, the exceptional sensitivity of the $\mathrm{HBr}$ DEA cross section at $0.3 \mathrm{eV}$ can be understood by looking at particular DEA cross sections for given angular momenta $J$. In Fig. 2 we plot the cross section for $J$ in the range $J=8-14$. When increasing $J$ from 8 to 9 the cross section changes significantly. The $J=9$ cross section is extremely narrow and its magnitude is four times higher than that for $J=8$. At higher values of $J$ the cross section remains almost constant but the corresponding widths broaden strongly. The width of the narrow $J=9$ peak is a very sensitive function of the parameters of the model.

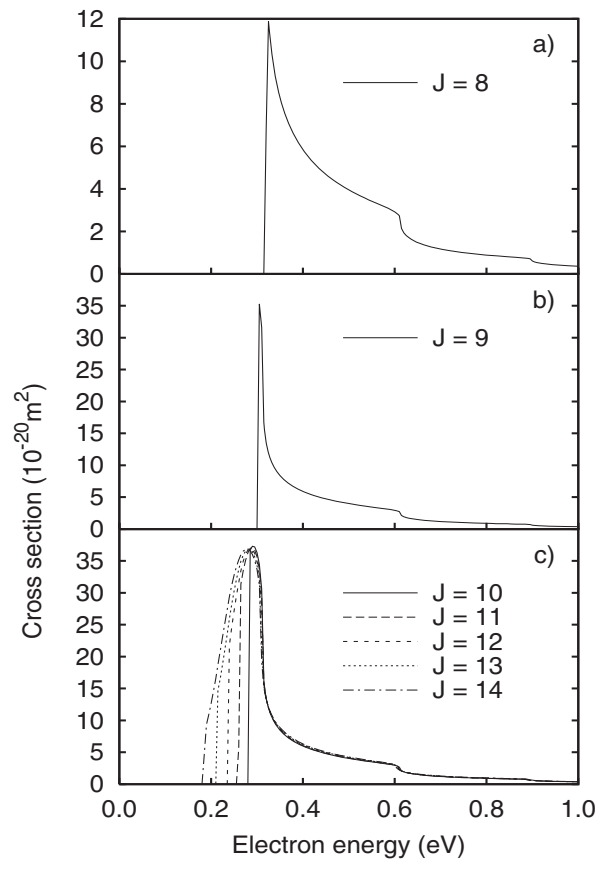

FIG. 2. DEA cross section for various angular momenta $J$, $v=0$. The cross section for $J=8$ is much smaller and significantly broader than that for $J=9$.

Figure 3 shows the measured $\mathrm{Br}^{-}$signal as a function of the incident electron energy and a comparison with earlier data and the present calculations. The predicted growth of the $0.3 \mathrm{eV}$ peak and the appearance of near-zero energy structures with the increasing temperature can be clearly observed in the present data.

The spectrum taken at $310 \mathrm{~K}$ is compared with the measurements of Allan and co-workers [4] and Abouaf and Teillet-Billy [6] (top panel Fig. 3). The temperature in the measurements of Allan was $310 \mathrm{~K}$, Abouaf and Teillet-Billy do not specify the gas temperature in their experiment. The somewhat narrower main DEA peak and the clearer resolution of the Wigner cusps in the spectrum of Allan and coworkers as compared to the present one can be ascribed to the better electron energy resolution in their experiment. However, this does not account for the lower relative intensity of the $0.3 \mathrm{eV}$ peak. It is important to point out that we had to shift our data and the spectrum of Abouaf et al. to higher energies by $30 \mathrm{meV}$ so that the positions of the resonances agreed with data of Allan.

This matching procedure is necessary if different energy calibration procedures have been invoked. Allan and coworkers [3] calibrated the energy scale using the $\mathrm{He}^{-}\left({ }^{2} \mathrm{~S}\right)$ negative ion scattering resonance at $19.366 \mathrm{eV}$. Abouaf and Teillet-Billy used the onset of $\mathrm{O}^{-} / \mathrm{CO}$ cross section estimating a $40 \mathrm{meV}$ uncertainty in their calibration. As mentioned above we calibrated the energy scale using the $s$-wave zero energy resonance in $\mathrm{SF}_{6}^{-}$. When calibrating with zero-energy peaks in crossed-beam experiments two effects have to be taken into account $[35,36]$ when comparing data obtained by other calibration procedures. First, the convolution integral in the region around the zero electron energy is not symmetric, see Eq. (3). Second, the abundance of the very low en- 


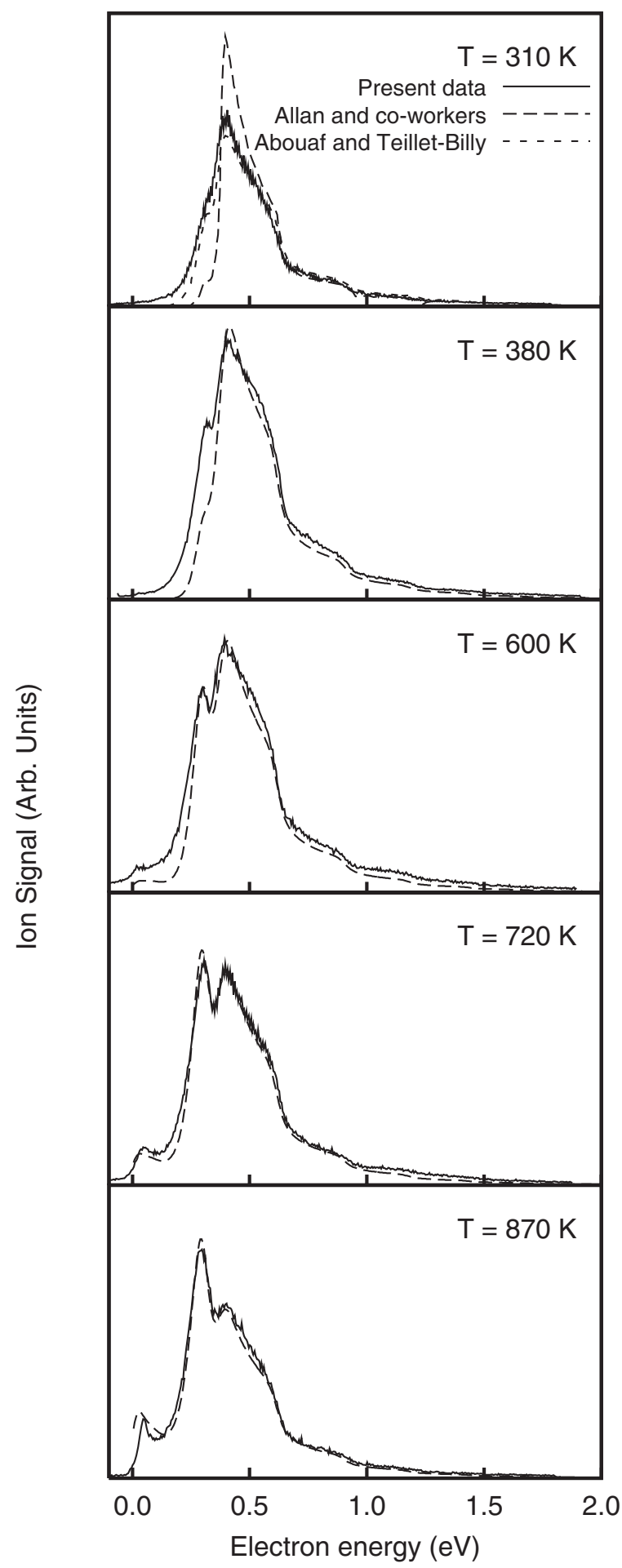

FIG. 3. Top panel: Comparison of the present ion yield spectrum at $310 \mathrm{~K}$ with the data of Allan and co-workers [4] and Abouaf and Teillet-Billy [6]. The present data and the spectrum of Aboauf were shifted to higher energies by $30 \mathrm{meV}$, see text. Lower panels: Comparison of the theoretical DEA cross section (dashed line) with the experimental data (full line). The theoretical data are convoluted with a Gaussian of $50 \mathrm{meV}$ FWHM. The experimental data are shifted to higher energies by $30 \mathrm{meV}$. The intensities of the experimental and calculated spectra have been normalized to the same intensity of the broad peak. ergy electrons in the beam may be lower because these electrons are easily deflected from their proper paths even by weak stray electrostatic fields. Both effects tend to shift the apparent maximum of the measured $\mathrm{SF}_{6}^{-}$peak to higher energies compared to the "correct" zero energy position. We conclude, that the $30 \mathrm{meV}$ difference between the data of Allan and our data is the right order of magnitude caused by these effects.

As mentioned above theoretical cross sections shown in Fig. 3 were convoluted with a Gaussian resolution function of $50 \mathrm{meV}$ FWHM. We observe a perfect agreement between the position of the resonances as well as between their relative height for the temperatures above $600 \mathrm{~K}$. However, the calculations underestimate the relative intensity of the $0.3 \mathrm{eV}$ resonance at temperatures up to $380 \mathrm{~K}$. Similarly, at $600 \mathrm{~K}$ the experimental cross section is slightly larger in the region around $0 \mathrm{eV}$.

In order to elucidate the observed difference in the intensities of the peaks at different temperatures, we have varied several parameters in the model of Č́žzek et al. [4]. In particular, in Ref. [4] the Morse potential curve based on parameters from Refs. [37] was used as a potential of the neutral molecule $V_{0}(R)$. The high quality ab initio data for $V_{0}(R)$ by Leininger and Gadea were presented in Ref. [4]. However, they were not used in the model due to disagreement of the calculated $\mathrm{HBr}$ dissociation energy and the experimental one. The difference was approximately $100 \mathrm{meV}$. This leads to an incorrect dissociative attachment threshold calculated using the $a b$ initio potential. Thus we modified the asymptotic part of the $a b$ initio potential to get the correct asymptotic value. From this $V_{0}$ we constructed the discrete state potential $V_{d}$ and the coupling matrix elements $V_{d \epsilon}$. The DEA cross sections calculated using this modified nonlocal resonance model differed from the predictions of the original model only negligibly. Additionally we have tried different shapes of the neutral $\mathrm{HBr}$ potential (keeping the dissociation energy equal to that of Ref. [37]), similarly they did not change the calculated cross sections significantly.

\section{CONCLUSIONS}

In conclusion, the present experiments provide accurate data for DEA to $\mathrm{HBr}$ for a range of temperatures. The observed temperature effects (appearance of additional peaks at 0.3 and zero $\mathrm{eV}$ at higher temperatures) can be nicely described within the nonlocal resonance model. The reason for the relative higher abundance of the $0.3 \mathrm{eV}$ resonance as compared to the main peak in the experimental data below $380 \mathrm{~K}$ remains the only open question.

\section{ACKNOWLEDGMENTS}

The authors thank A. Stamatovic, Beograd, for extensive assistance in the laboratory. Work partially supported by FWF Wien, the Grant Agency of the Academy of Sciences of the Czech Republic grant no. IA100400501, and European Commission, Brussels, e.g., through the ESF EIPAM. 
[1] M. Čížek, J. Horáček, M. Allan, I. I. Fabrikant, and W. Domcke, J. Phys. B 36, 2837 (2003).

[2] A. Ch. Sergenton, L. Jungo, and M. Allan, Phys. Rev. A 61, 062702 (2000).

[3] M. Č́ižek, J. Horáček, and W. Domcke, Phys. Rev. A 60, 2873 (1999).

[4] M. Čížek, J. Horáček, A. Ch. Sergenton, D. B. Popović, M. Allan, W. Domcke, T. Leininger, and F. X. Gadea, Phys. Rev. A 63, 062710 (2001).

[5] J. P. Ziesel, I. Nenner, and G. J. Schulz, J. Chem. Phys. 63, 1943 (1975).

[6] R. Abouaf and D. Teillet-Billy, Chem. Phys. Lett. 73, 106 (1980).

[7] M. A. Morrison, Adv. At., Mol., Phys. 24, 51 (1988).

[8] I. I. Fabrikant, Comments At. Mol. Phys. 24, 37 (1990).

[9] W. Domcke, Phys. Rep. 208, 97 (1991).

[10] J. Horáček, in The Physics of Electronic and Atomic Collisions, XXI ICPEAC, Sendai, edited by Y. Itikawa et al. (American Institute of Physics, New York, 1999), p. 329.

[11] G. J. Schulz, Rev. Mod. Phys. 45, 423 (1973).

[12] H. S. W. Massey, Negative Ions (Cambridge University Press, Cambridge, 1976).

[13] T. F. O’Malley, Phys. Rev. 155, 59 (1967).

[14] W. R. Henderson, W. L. Fite, and R. T. Brackmann, Phys. Rev. 183, 157 (1969).

[15] D. Spence and G. J. Schulz, Phys. Rev. 188, 280 (1969).

[16] M. Allan and S. F. Wong, Phys. Rev. Lett. 41, 1791 (1978).

[17] J. M Wahedra and J. N. Bardsley, Phys. Rev. Lett. 41, 1795 (1978).

[18] D. E. Atems and J. M. Wadehra, Phys. Rev. A 42, 5201 (1990)

[19] A. P. Hickman, Phys. Rev. A 43, 3495 (1991).

[20] Y. Xu, G. A. Gallup, and I. I. Fabrikant, Phys. Rev. A 61,
052705 (2000).

[21] M. Allan and S. F. Wong, J. Chem. Phys. 74, 1687 (1981).

[22] M. Braun, F. Gruber, M. W. Ruf, S. V. K. Kumar, E. Illenberger, and H. Hotop, Chem. Phys. 329, 148 (2006).

[23] M. Fenzlaff, R. Gerhard, and E. Illenberger, J. Chem. Phys. 88, 149 (1988).

[24] P. G. Datskos and L. G. Christophorou, J. Chem. Phys. 90, 2626 (1989).

[25] I. Hahndorf and E. Illenberger, Int. J. Mass Spectrom. Ion Process. 167/168, 87 (1997).

[26] R. S. Wilde, G. A. Gallup, and I. I. Fabrikant, J. Phys. B 32, 663 (1999).

[27] S. Matejcik, I. Ipolyi, and E. Illenberger, Chem. Phys. Lett. 375, 660 (2003).

[28] M. Külz, M. Keil, A. Kortyna, B. Schellhaass, J. Hauck, K. Bergmann, W. Meyer, and D. Weyh, Phys. Rev. A 53, 3324 (1996).

[29] S. Živanov, M. Allan, M. Čížek, J. Horáček, F. A. U. Thiel, and H. Hotop, Phys. Rev. Lett. 89, 073201 (2002).

[30] V. Grill, H. Drexel, W. Sailer, M. Lezius, and T. D. Märk, Int. J. Mass. Spectrom. 205, 209 (2001).

[31] For instance, J. F. O'Hanlon, A User's Guide to Vacuum Technology (Wiley, Hoboken, NJ, 2003).

[32] D. M. Bishop and L. M. Cheung, J. Phys. Chem. Ref. Data 11, 119 (1982).

[33] H. J. Werner and P. Rosmus, J. Chem. Phys. 73, 2319 (1980).

[34] M. Braun, S. Barsotti, S. Marienfeld, E. Leber, J. M. Weber, M. W. Ruf, and H. Hotop, Eur. Phys. J. D 35, 177 (2005).

[35] A. Stamatovic (private communication).

[36] P. D. Burrow (private communication).

[37] K. P. Huber and G. Herzberg, Molecular Spectra and Molecular Structure (Van Nostrand, New York, 1979), Vol. IV. 